\title{
Echocardiographic guidance of pulmonary vein isolation catheter ablation procedure for recurrent atrial fibrillation in partial cor triatriatum
}

\author{
Bernard P. Paelinck ${ }^{1,2}$, Paul L. Van Herck ${ }^{1}$, Lien Vandaele ${ }^{1}$, Andrea Sarkozy ${ }^{1}$ \\ ${ }^{1}$ Department of Cardiology, University Hospital Antwerp, Edegem, Belgium \\ 2Department of Cardiac Surgery, University Hospital Antwerp, Edegem, Belgium
}

A 70-year-old man was admitted for pulmonary vein isolation (PVI) with catheter ablation because of recurrent symptomatic atrial fibrillation (AF). Our preprocedural imaging algorithm includes a routine computed tomography scan. The scan revealed partial cor triatriatum sinister (Fig. 1A, B). During PVI, three-dimensional transoesophageal echocardiography (3D TEE) guidance showed a complex triangular left atrial membrane adherent to the interatrial septum, located anteriorly from the right inferior pulmonary vein (PV) and extending behind the non-coronary cusp (Fig. 1C). Colour Doppler did not demonstrate flow in the tunnel-shaped structure or flow obstruction in the left atrium. To avoid puncture through the left atrial membrane, 3D TEE guidance was used to puncture the interatrial septum posterior to the membrane (Fig. 1D, Suppl. Video 1 and 2 - see journal website). An electroanatomic map of the left atrium was created, and the membrane was tagged using a contact force-sensing radiofrequency ablation catheter (Fig. 1E) and 3D TEE guidance. Circumferential PVI was performed with the ablation line around the right PVs, designed to run posterior to the membrane at the anterior antrum (Fig. 1E). Right and left PV electrical isolation was successful. The absence of entry and exit blocks
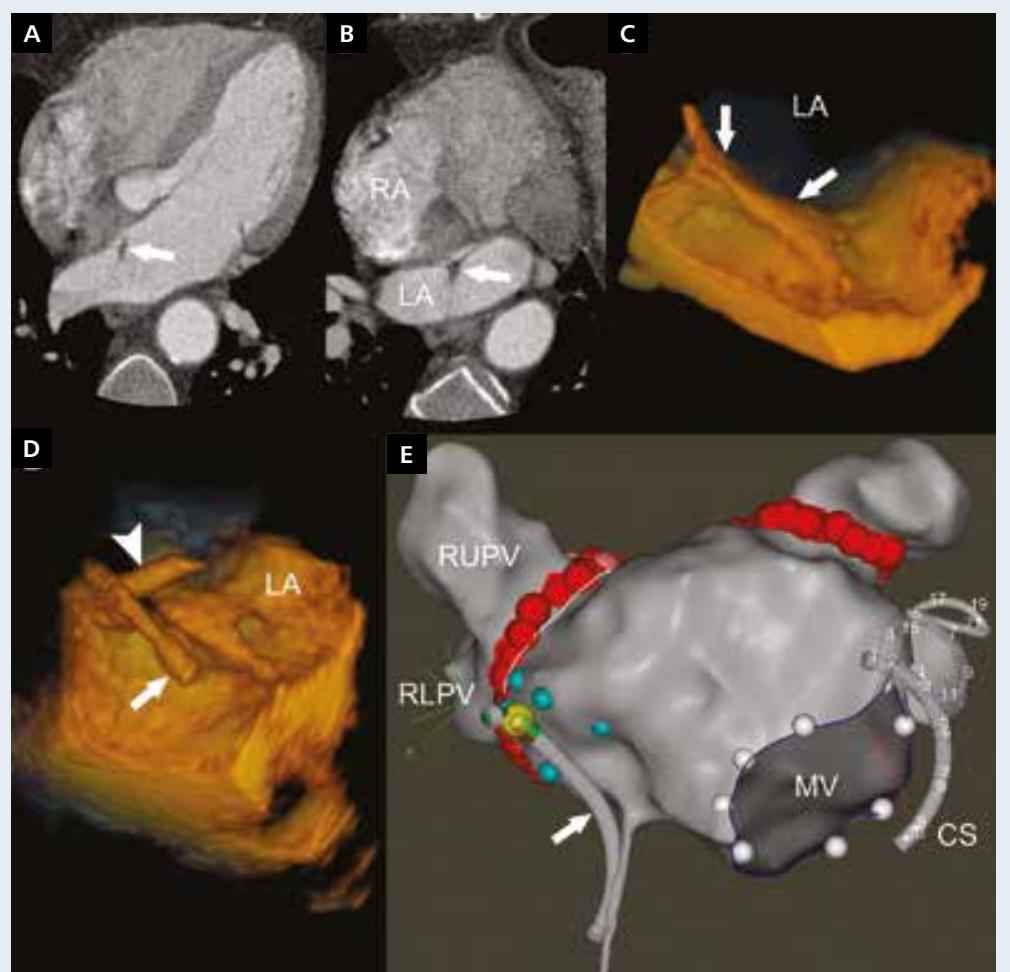

Figure 1. Computed tomography scan (A, B) and three-dimensional transoesophageal echocardiography (C, D) of complex triangular left atrial membrane (white arrows) adherent to the interatrial septum; LA — left atrium; RA — right atrium; D. White arrow: ablation catheter; white arrowhead: second transseptal mapping catheter; E. Electroanatomic map of the LA and tagging of the membrane; blue tags; CS - coronary sinus catheter; MV — mitral valve; RLPV — right lower pulmonary vein; RUPV — right upper pulmonary vein was confirmed by adenosine testing. The post-ablation course remained uneventful. Seven months after the PVI, the patient was free of AF. Cor triatriatum sinister is a rare developmental anomaly resulting in a fibromuscular septum dividing the left atrium. It often presents as a mitral stenosis-like syndrome and AF [1]. The therapeutic options for atrial flow obstruction include surgical resection or transcatheter balloon dilatation of the membrane [1]. There have been limited reports of $\mathrm{PVI}$ in cor triatriatum sinister [2]. Partial cor triatriatum sinister may be an incidental finding in adults, presenting as AF [3]. 3D TEE is crucial for a safe PVI procedure in complex atrial anatomy.

\section{References}

1. Patel MB, Samuel BP, Berjaoui WK, et al. Transcatheter intervention in cor triatriatum sinister. Can J Cardiol. 2015; 31(6): 819.e3-819.e4, doi: 10.1016/j. cjca.2015.01.036, indexed in Pubmed: 26022992.

2. Gavin A, Singleton CB, McGavigan AD. Successful multi-chamber catheter ablation of persistent atrial fibrillation in cor triatriatum sinister. Indian Pacing Electrophysiol J. 2011; 11(2): 50-55, indexed in Pubmed: 21468249 .

3. Ker J. Cor triatriatum sinister presenting with adult onset atrial fibrillation-another rare cause for a common clinical problem. Int J Cardiol. 2013; 167(1): e12-e13, doi: 10.1016/j.ijcard.2013.01.269, indexed in Pubmed: 23465227.

\section{Address for correspondence:}

Bernard P. Paelinck, MD, PhD, Departments of Cardiology Cardiac Surgery, University Hospital Antwerp, Wilrijkstraat 10, 2650 Edegem, Belgium, tel: +32 3/8214182, fax: +32 3/8250848, e-mail: bernard.paelinck@uza.be

Conflict of interest: none declared

Kardiologia Polska Copyright @ Polish Cardiac Society 2018 